\title{
An impact of reduction in point prevalence of tobacco use on cancer incidence- A challenge for global policy makers
}

\author{
Dr Atanu Bhattacharjee ${ }^{\mathrm{a}}$, Dr Subita Patil ${ }^{\mathrm{b}, *}$, Mr Sanjay Talole $^{\mathrm{c}}$, Dr Arjun Singh ${ }^{\mathrm{d}}$, \\ Dr Pankaj Chaturvedi ${ }^{\mathrm{e}}$, Dr Rajesh Dikshit ${ }^{\mathrm{f}}$ \\ ${ }^{a}$ Asst. Prof., Sec of Biostatistics, Centre for Cancer Epidemiology, Tata Memorial Centre, Homi Bhabha National Institute, Mumbai, India \\ ${ }^{\mathrm{b}}$ Physician, Dept of Preventive Oncology, Centre for Cancer Epidemiology, Tata Memorial Hospital, Tata Memorial Centre, Homi Bhabha National Institute, India \\ ${ }^{c}$ Scientific Officer-E, Sec of Biostatistics, Cancer Centre of Epidemiology, Tata Memorial Centre, India \\ ${ }^{\mathrm{d}}$ Senior Research Fellow, Department of Head Neck Surgery, Tata Memorial Hospital, India \\ ${ }^{\mathrm{e}}$ Prof \& Surgeon, Department of Head Neck Surgery, Tata Memorial Hospital, Deputy Director, Centre for Cancer Epidemiology, Tata Memorial Centre, Homi Bhabha \\ National Institute, Mumbai, India \\ ${ }^{\mathrm{f}}$ Director, Centre for Cancer Epidemiology, Tata Memorial Centre, Homi Bhabha National Institute, Mumbai, India
}

\section{A R T I C L E I N F O}

\section{Keywords:}

Tobacco prevalence

Tobacco related cancers

Cancer incidence

\begin{abstract}
A B S T R A C T
Worldwide 8.2 million people die from cancer \& 2/3rd of them are from LMIC. Tobacco-related cancer (TRCs) accounts a major share. In India, $45 \%$ of male's and $20 \%$ of female's cancer is due to tobacco use. Nearly half of all cancers in men occur at sites associated with tobacco use( Farhood and et al., 2018 Mar) .11

Objective: This attempt to find out impact of tobacco prevalence on incidence rate of Tobacco related cancers across various states of India. This can help policy makers to understand and formulate control measures to curb the increasing trend of TRCs.

Methods: Age standardised incidence rate of TRCs from Global Burden of Diseases, Injuries, and Risk Factors Study and tobacco prevalence from NFHS-4 data was observed. State-wise data harmonisation towards tobacco prevalence and cancer incidence was performed.

Results: Reduction of tobacco consumption prevalence only 1\% may decline incidence of lip, oral cavity and larynx cancer by 23.56 per 10 lac female populations and incidence of pharynx cancer by 25.31 per 10 lac male populations.

Conclusion: Tobacco consumption contributes substantial AAR of TRCs among both sexes in most of the Indian states while, other factors need to be considered too. Hence, systematic, multi-sectorial coercive approach to curtail the burden related to TRCs is imperative.
\end{abstract}

\section{Introduction}

According to WHO, every year across the world 8.2 million people die from cancer and two-thirds of these deaths occur in low and middle income countries. (LMIC) ${ }^{1}$ Worldwide, deaths from cancer are predicted to upsurge more than 13.1 million by $2030 .^{2}$ GLOBOCAN estimates about 14 million new cancer cases were diagnosed worldwide in 2012; One million of these new cases and nearly 7 lakh deaths occurred in India. ${ }^{3}$ Tobacco use, in any form, accounts for $22 \%$ of cancer deaths globally, and is a leading cause of the disease in the South-East Asia Region. However, more than $50 \%$ of deaths can be prevented. ${ }^{4}$ Seventeen million new cases are expected worldwide by 2020 with threefourth of the cancer-related deaths occurring in developing countries of the world. Out of all cancers, tobacco-related cancer (TRC) accounts a major share. WHO estimates that one out of two young people who start smoking and continue smoking throughout their lives will lead to TRCs. ${ }^{5}$ To the best of our knowledge, we could not find any community based study which could elaborate the linkage between tobacco prevalence and cancer incidence globally.

In India, tobacco added a major burden on the total cancer cases and nearly $45 \%$ of male's cancer and $20 \%$ of female's cancer is due to tobacco use. ${ }^{6}$ India also has one of the highest rates of oral cancer in the world, partly attributed to high prevalence of tobacco chewing. ${ }^{7}$ The proportion of TRCs varies in India according to the geographical region. ${ }^{6}$ Nearly half of all cancers in men occur at sites associated with tobacco use. While In women, less than one fifth of cancers occur at

\footnotetext{
* Corresponding author.

E-mail addresses: atanustat@gmail.com (D.A. Bhattacharjee), psubita@gmail.com (D.S. Patil), sdtalole@gmail.com (M.S. Talole), arjun193@gmail.com (D.A. Singh), chaturvedi.pankaj@gmail.com (D.P. Chaturvedi), dixr24@hotmail.com (D.R. Dikshit).
} 
sites, predominantly in esophagus and oral cavity, but cancers of the cervix and breast constitutes over $40 \%$ of all cancers. ${ }^{7}$ The lower proportion of tobacco related cancers (TRCs) among women is mostly explained by the fact that, tobacco use, especially smoking, is relatively not common among women. ${ }^{8}$

We were fortunate to have data from community based national survey on tobacco consumption prevalence from National Family Health Survey -IV (year 2015-16) and also data on cancer incidence rate published in Global Burden of Diseases, Injuries, and Risk Factors Study (GBD) conducted in year 2016. This data was not synchronized till date. This is an attempt to find out impact of tobacco prevalence on incidence rate of Tobacco related cancers across various states of India. This can help policy makers to understand and formulate various tobacco control measures to curtail the increasing disease trend of TRCs globally.

\section{Objective}

To find out impact of tobacco use on incidence of Tobacco related cancers across various states of India.

\section{Methods}

(Refer Fig. 1: Flowchart of methodology).

The present study observed data on tobacco related cancers from Global Burden of Diseases Injuries, and Risk Factors Study. ${ }^{9}$ The India State-Level Disease Burden Initiative was a collaboration with the Global Burden of Diseases, Injuries, and Risk Factors Study (GBD) to produce subnationalz disease burden estimates for India. It was published by Dandona et al. ${ }^{9}$ in Oct 2018, Lancet. The Global Burden of Diseases (GBD) methods depend on significantly on the National Cancer Registry Programme (NCRP), India. Also, considers data during year 1990-2016 from all accessible data sources from some registries, not included under NCRP, cause of death data from the Sample Registration System (SRS) and other sources. ${ }^{9}$ NCRP descriptive Cancer Epidemiology Report covers 23 states \& union territories.

Tobacco Related Cancer (TRC) sites (along with International Classification of Diseases code): lip (C00), tongue (C01-C02), mouth (C03-C06), oropharynx (C10), hypopharynx (C12-C13), pharynx (C14), esophagus (C15), larynx (C32), lung (C33-C34), and urinary bladder (C67). But data on following TRCs were available in GBD study was-lip \& oral cavity, pharynx, nasopharynx, larynx, lung and urinary bladder cancer. We considered Incidence rate (per 10, 00, 000 population)of above mentioned cancers for the burden of disease. ${ }^{9}$ We considered age adjusted incidence rate (AAR) as it allow comparisons over time $\&$ between states after adjusting for the differences in the age

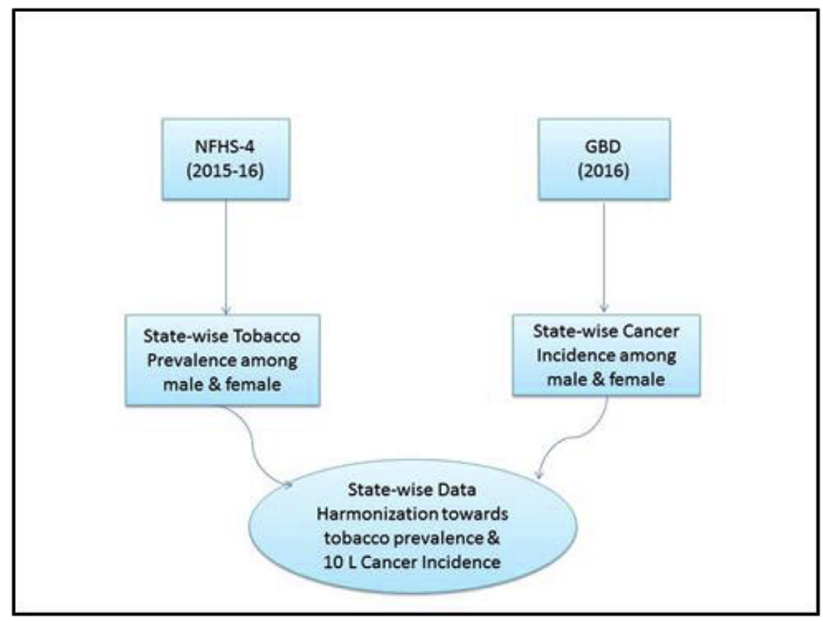

Fig. 1. Methodology flow chart. structure of the population. ${ }^{9}$

We used data from the National Family Health Survey-IV ${ }^{10}$ conducted in year 2015-16 to examine the state-wise distribution of tobacco consumption in the form of prevalence of tobacco was considered.

The National Family Health Survey (NFHS) was a large-scale, multiround survey conducted in a representative sample of households throughout India. ${ }^{10}$ Four rounds of NFHS were conducted in the following years: 1992-93, 1998-99, 2005-06 and in 2015-16. NFHS-4 fieldwork for India was conducted from January 20, 2015 to December 4, 2016 by 14 Field Agencies and gathered information from 601,509 households, 699,686 women, and 112,122 men.

Afterwards, Indian states were arranged in descending order with respect to incidence rate of cancers. First four Indian states having highest incidence of cancer and last four Indian states having lowest incidence rate for that cancer was arranged. Average of each was calculated. The difference between average of high and average of low incidence rate for cancer was calculated.

Similarly, average of tobacco prevalence for those states having high incidence and low incidence of cancer was calculated. The difference between average of high \& low tobacco prevalence was obtained.

It was done for above mentioned all six TRCs, for male and female separately.

\section{Results}

According to National Family Health Survey-IV, 44.5\% of men and $6.8 \%$ of women aged 15-49 years, use some form of tobacco. Refer Table: 1 . Use of any kind of tobacco has decreased from 57 to $44.5 \%$ among men and 10.8 to $6.8 \%$ among women in last one decade. ${ }^{11}$ There was $12.5 \%$ and $4 \%$ decline in tobacco consumption among men \& women respectively. Paan masala/gutkha were the most common form of tobacco consumption among both sexes in India. Among men this was followed by cigarette/bidi smoking.

Hence, we plotted tobacco consumption prevalence among male \& female separately for all states \& UT of India to observe state variation. We considered AAR for TRCs from GBD study state-wise (year 2016). Thus, we plotted line diagramme which showed relationship of different TRCs and tobacco consumption prevalence among male \& female.

The use of tobacco \& AAR of lip \& oral cavity cancer were directly associated among males as showed in Fig. 2. Following states had high AAR Ca lip \& oral cavity among males: Gujarat, Madhya Pradesh, Meghalaya, UT other than Delhi. According to NFHS-IV data Gujarat had $51.4 \%$ of tobacco prevalence but NFHS-III data had prevalence of $60.2 \%$ among males. Indicating that high tobacco use a decade ago caused increased lip \& oral cavity cancer among males of Gujarat presently. High prevalence of male tobacco consumption was observed currently in Mizoram, Manipur, Nagaland \& Meghalaya.

Tobacco consumption \& AAR Ca lip \& oral cavity among females had parallel association with each other except Goa, Kerala where AAR was high irrespective of low tobacco prevalence. Refer Fig. 3.

Madhya Pradesh, UT (Other than Delhi), Karnataka \& Odissha had highest AAR Ca lip \& oral cavity among females. Easy availability of tobacco in home due to spouse use can explain high incidence of CA lip \& oral cavity among both sexes in Madhya Pradesh, UT other than Delhi. Currently, Odissha had female tobacco consumption as $17.3 \%$ but had reduced by $14.1 \%$ in last decade. To get the effect of reduced tobacco consumption, one needs to observe the trend of that cancer over a period. Presently, high no. of lip \& oral cavity CA among females explains the fact that previous use will give the effect over a period of time.

Tobacco consumption up surged AAR Ca larynx among males as depicted in Fig. 4. Gujarat, Madhya Pradesh, Meghalaya, UT other than Delhi had high number of cancer larynx and had high prevalence of 
Table: 1

Tobacco consumption among adults (age 15-49 years).

\begin{tabular}{|c|c|c|c|c|c|c|}
\hline & & \multicolumn{3}{|c|}{ NFHS-4 (2015-16) } & \multirow[t]{2}{*}{ NFHS-3 (2005-06) } & \multirow[t]{2}{*}{ Decline in use } \\
\hline & & Urban & Rural & Total & & \\
\hline 1 & Women who use any kind of tobacco (\%) & 4.4 & 8.1 & 6.8 & 10.8 & $4 \%$ \\
\hline 2 & Men who use any kind of tobacco (\%) & 38.9 & 48.0 & 44.5 & 57.0 & $12.5 \%$ \\
\hline 3 & Women who tried to stop smoking or using tobacco in any other form during the past 12 months(\%) & 33.0 & 28.2 & 29.3 & $\mathrm{Na}$ & \\
\hline 4 & Men who tried to stop smoking or using tobacco in any other form (during the past 12 months)(\%) & 29.6 & 31 & 30.6 & $\mathrm{Na}$ & \\
\hline
\end{tabular}

tobacco consumption. Madhya Pradesh had high larynx cancer in both sexes (Refer Figs. 4 and 5). Ca lip \& oral cavity \& larynx were high in Gujarat, Madhya Pradesh, Meghalaya \& UT other than Delhi. All these states had high prevalence of male tobacco consumption ranging between 51 and $72 \%$. Males from Meghalaya having high consumption of tobacco had highest number of lip, oral cavity \& larynx cancer. In Meghalaya, male tobacco prevalence had increased by $2.9 \%$ since last decade which alarms increased tobacco related cancer incidence burden.(NFHS-3: $69.3 \%$ to NFHS-4: 72.2\%).

Tobacco use and AAR Ca larynx among females were related to each other as shown in Fig. 5. But irrespective of average tobacco consumption among females in Madhya Pradesh, UT (other than Delhi), Karnataka \& Odissha, there was high AAR Ca lip \& oral cavity, larynx.

Regardless of wide variation of tobacco prevalence among males of various states there was no rise in AAR Ca nasopharynx, exception was Nagaland as indicated in Fig. 6. The male tobacco prevalence had increased by $1.3 \%$ (NFHS-3: $67.9 \%$ to NFHS-4: $69.2 \%$ ) since last decade and had highest number of AAR Ca nasopharynx cancer among male. Bidi smoking was common along with smokeless tobacco form in NorthEast region. In Mizoram, nasopharyngeal cancer was second in occurrence while first common was lung. Similar findings observed among males from Manipur. Similar findings were noted for females except Tripura where irrespective of high tobacco consumption, AAR Ca nasopharynx was low. Female nasopharnyx cancer was observed high among states having high tobacco consumption: Mizoram, Arunachal Pradesh, Nagaland \& Sikkim. Refer Fig. 7.
Tobacco prevalence contributes higher AAR Ca pharynx among males was revealed in Fig. 8. Meghalaya, Aasam and Rajasthan had high Pharynx cancer and high tobacco consumption prevalence among males. Harayana showed high incidence for pharynx cancer among both sexes. Prevalence of tobacco consumption among males from Harayana was average while among females (Ref Fig. 9) very less, still Ca pharynx was high among both sexes. This needs more learning \& research of other etiological factors. Rajasthan had high no. of AAR Ca Pharynx among both sexes. Prevalence of Tobacco consumption was high among males from Rajasthan but not among females. refer Fig. 9. Further studies need to be conducted to understand type/form, way of tobacco, duration \& frequency of use \& other etiological factors contributing to high number. Aassam, Meghalaya had high number of pharynx among TRCs because of high prevalence of tobacco use among males in those states. Tobacco prevalence among males at Haryana had decreased by $10.5 \%$ over last decade still high AAR was observed. The effect of decline in cancer incidence may take place over next decade. But among females tobacco prevalence was very less. Irrespective of this pharynx cancer was high which needs more research to undertake. Though there was less than $1 \%$ tobacco prevalence among female from Himachal Pradesh they had high incidence of Ca pharynx. Ref Fig. 9.

Tobacco consumption \& AAR Ca lung among males as directly proportional revealed in Fig. 10. AAR Ca lung was high among Mizoram, J\& K, Kerala, Manipur. Out of these states, Mizoram \& Manipur had high tobacco consumption among male. Manipur showed $1.2 \%$ rise in male tobacco consumption prevalence while $9.2 \%$ escalation among

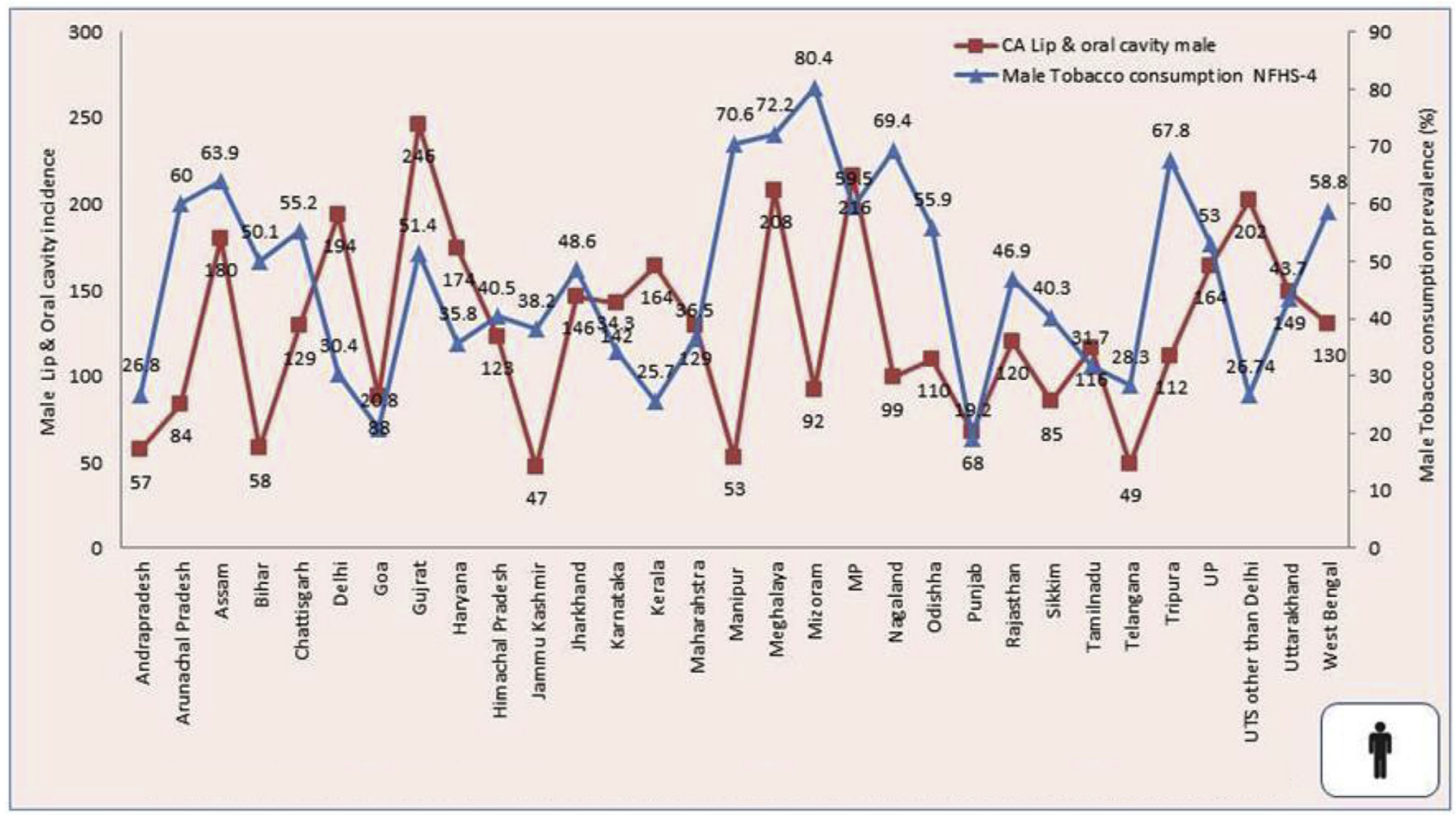

Fig. 2. Relationship between Tabacco prevalence (\%) and lip and oral cavity CA insidence (per 10L pop) among Male. 


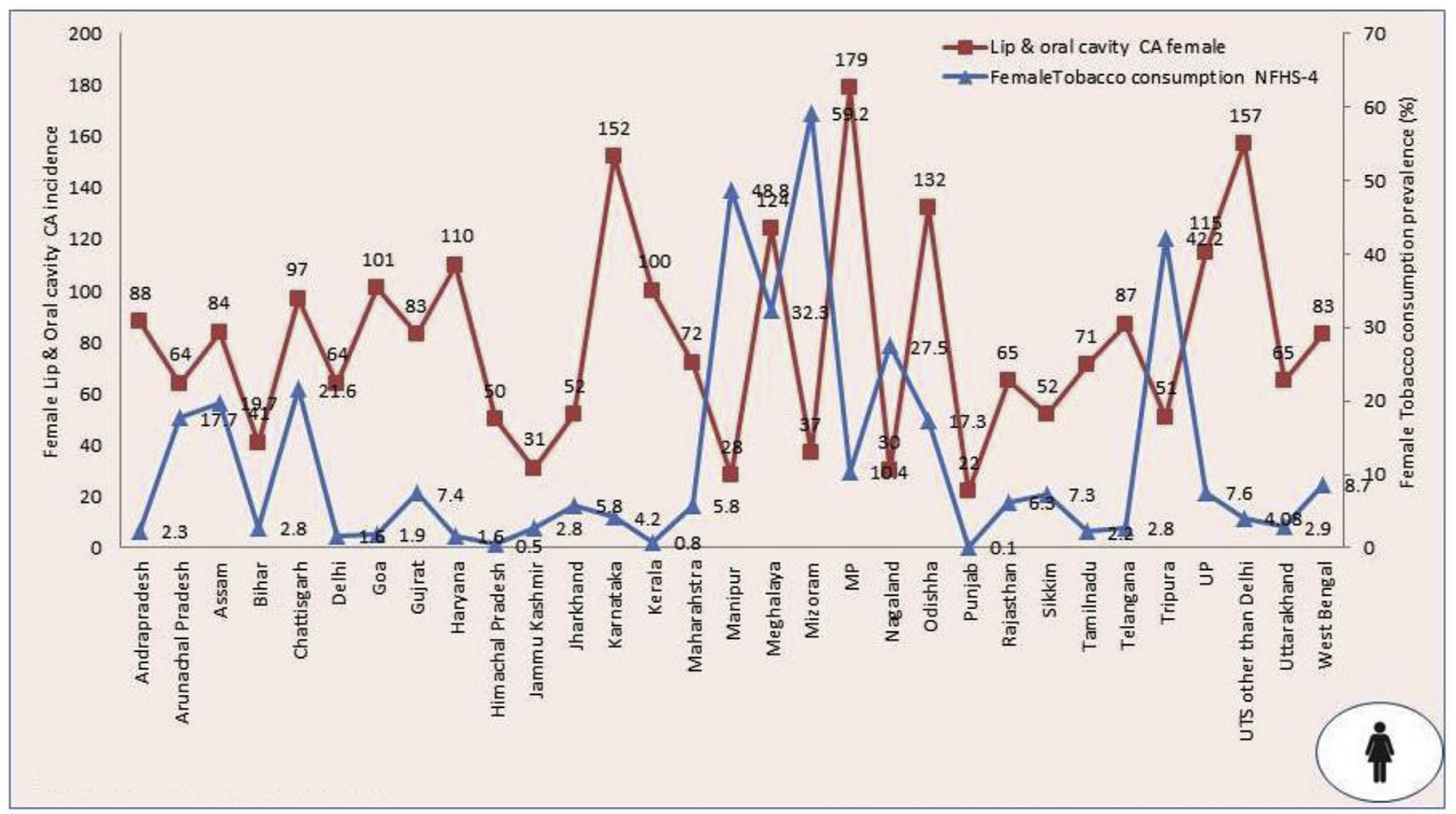

Fig. 3. Relationship between Tabacco consumption prevalence (\%) and lip and oral cavity CA insidence (per 10L pop) among Female.

females. There had been $17.8 \%$ reduction of tobacco consumption prevalence among Kerala men in last decade. Still lung cancer had high incidence among Kerala men. Because of commonest form of tobacco consumption being smoking, males were predisposed to lung cancer.

Similar findings among women were there like men with exception of few states for lung Ca. Female lung cancer was higher in Mizoram, Manipur, J \& K, and Sikkim. Refer to Fig. 11 Lung Ca was highest among both sexes in Mizoram, Manipur and J\& K. Peculiar form \& way of tobacco consumption inclined them for lung CA. Along with smoking, air pollution - indoor as well as outdoor pollution can be attributable for lung Ca.

Tobacco use \& AAR Ca bladder among males were directly related in most of the states as observed in Fig. 12. Delhi, Kerala, Chhattisgarh, Harayana had highest AAR Ca bladder. Tobacco consumption prevalence was average as per NFHS - 4 data among these states.

It was observed that bladder CA was higher among females at Chattisgarh, Delhi, Uttarakhand, UT (Other than Delhi). Refer Fig. 13. Except few states both variables were related to each other. Indicating along with tobacco consumption, other environmental factors affected incidence of Ca bladder. High tobacco consumption prevalence among

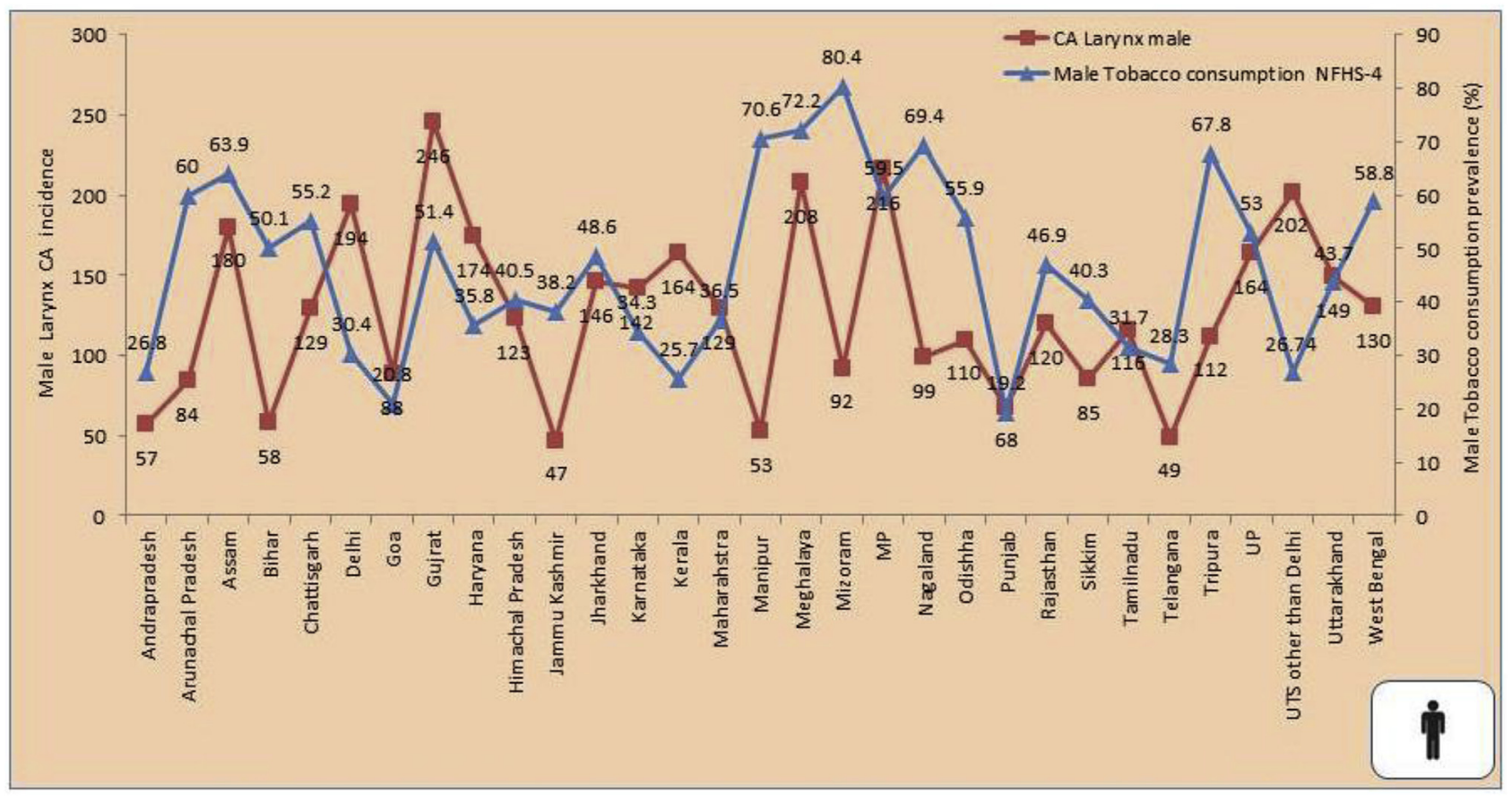

Fig. 4. Relationship between Tabacco prevalence (\%) and Larynx CA insidence (per 10L pop) among Male. 


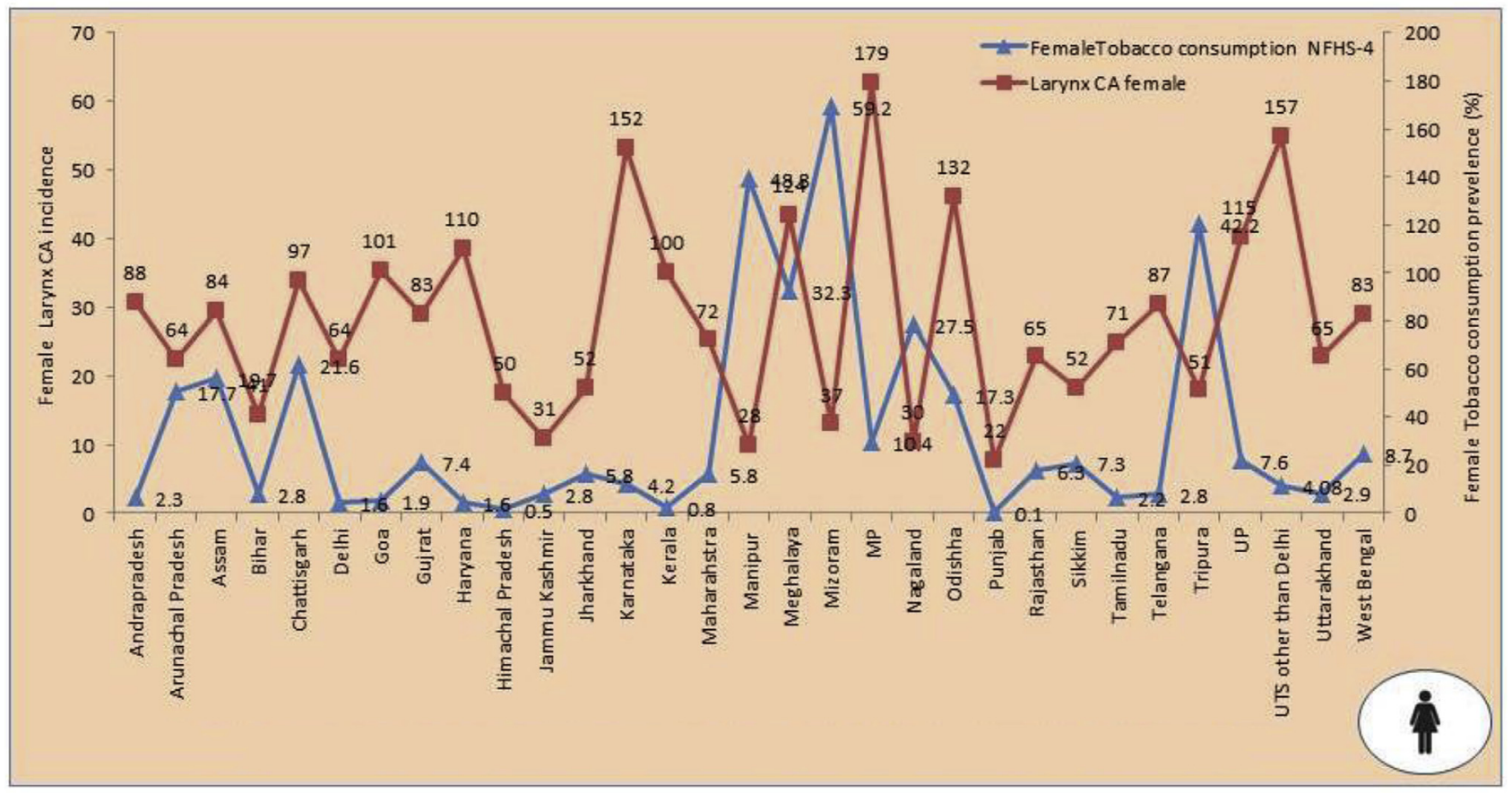

Fig. 5. Relationship between Tabacco prevalence (\%) and Larynx CA insidence (per 10L pop) among Female.

females in Manipur, Tripura \& Meghalaya did not show high incidence of bladder Ca. But Mizoram had high female tobacco prevalence and high female Ca bladder incidence rate.

We observed that tobacco consumption was attributable to incidence of TRCs majorly among various Indian states. However, consideration of other factors affecting TRCs incidence is also important. Those factors are genetic, geographic, dietary, lifestyle related, environmental, socioeconomic status, religion, culture, level of literacy, poverty, availability of health care facility, health seeking behaviour, and insurance facility. Also the type, form, way, frequency, duration of tobacco use, other habits, immunity does attribute the disease.
If one could reduce tobacco consumption prevalence to $1 \%$, cancer incidence can be curtailed as follows.

This work is dedicated only on specific TRCs. The linkage between tobacco prevalence and incidence rate of different types of cancer are observed. The state level tobacco prevalence with cancer incidence is explored. Further, the magnitude of site wise cancer incidence rate occurrence by single unit rate of tobacco prevalence appearance among male and female are drawn separately.(refer to point 3 methodology). As shown in Table 2, overall it shows that one unit reduction of tobacco prevalence may decline incidence of lip, oral cavity and larynx cancer among female by 23.56 per $1,000,000$. Similarly, maximum amount of

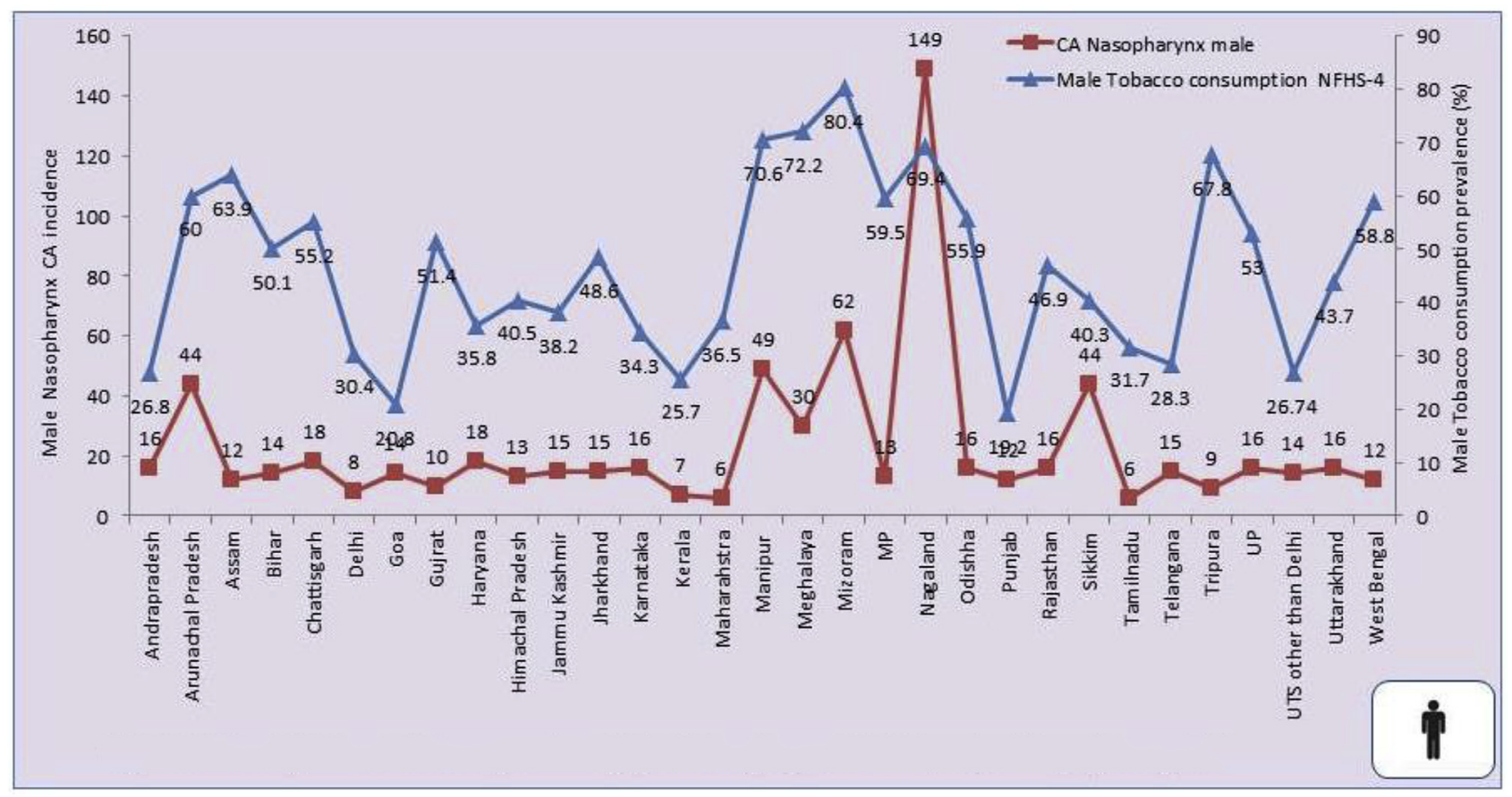

Fig. 6. Relationship between Tabacco prevalence (\%) and Nasopharynx CA insidence (per 10L pop) among Male. 


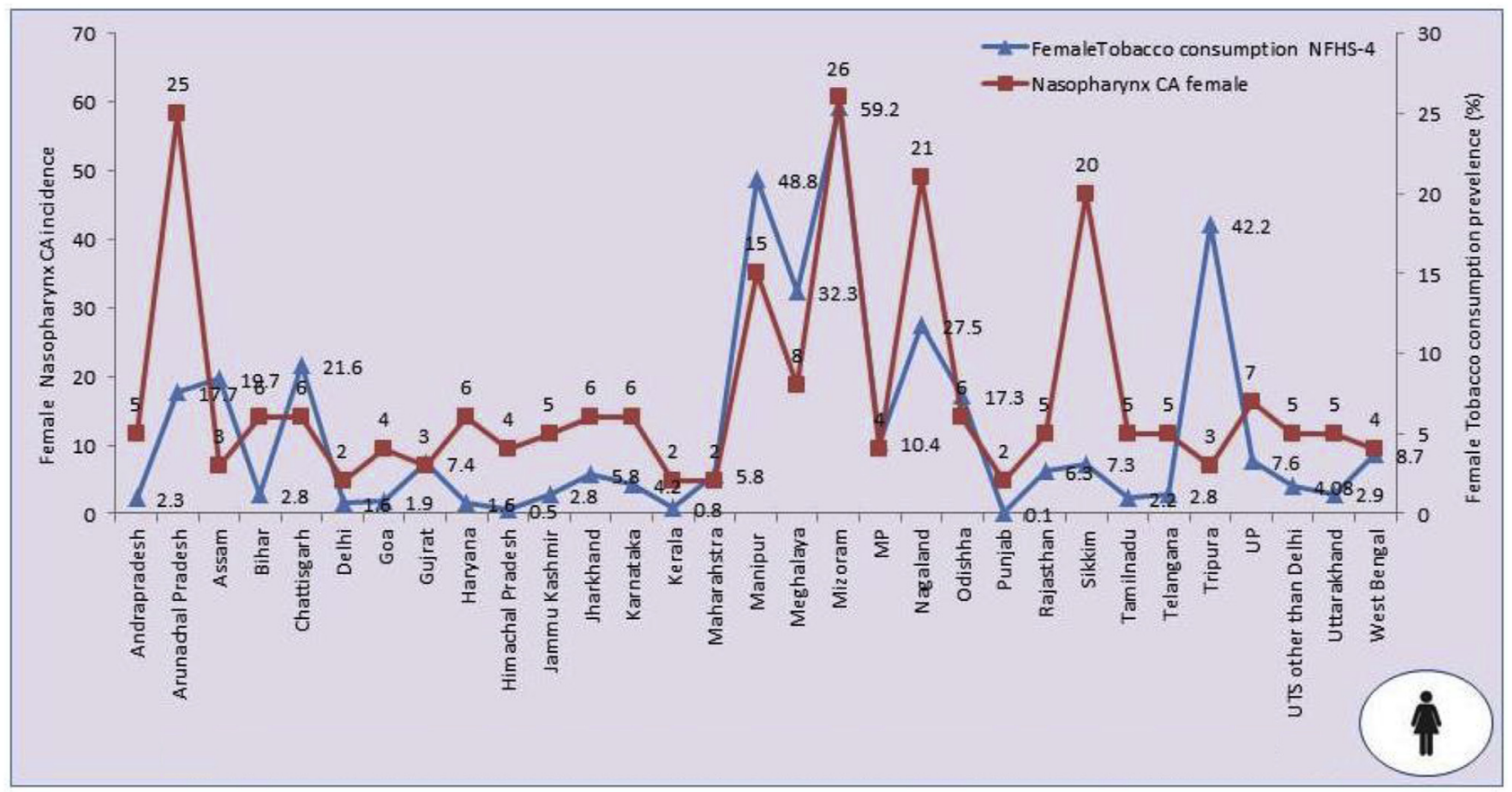

Fig. 7. Relationship between Tabacco prevalence (\%) and Nasopharynx CA insidence (per 10L pop) among Female.

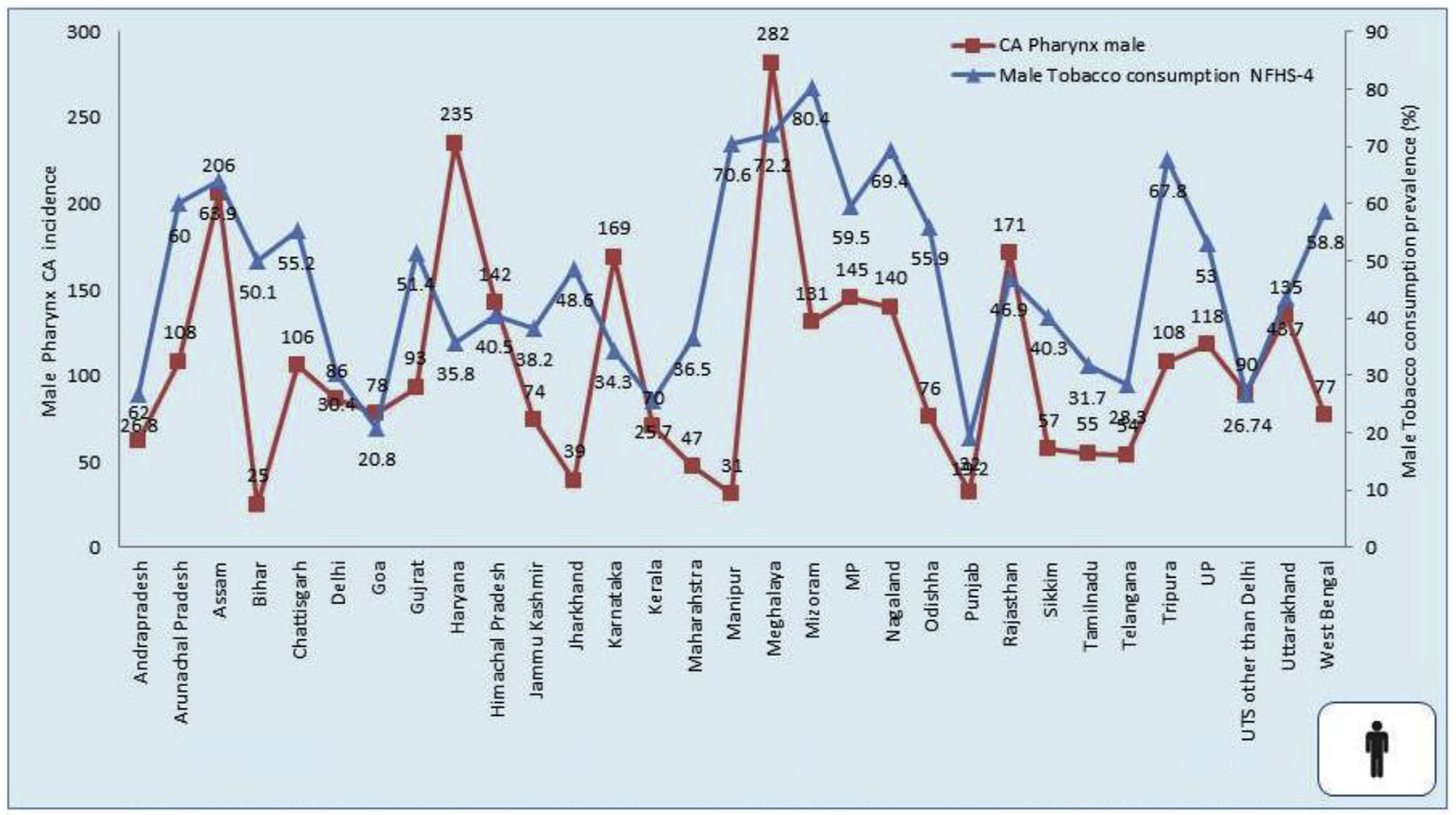

Fig. 8. Relationship between Tabacco consumption prevalence (\%) and pharynx CA insidence (per 10L pop) among Male.

pharynx cancer incidence i.e. 25.31 cases per 1,000,000 male population can be reduced if tobacco prevalence is reduced by single per cent in all Indian states. Gender wise and site wise linkage are provided in Table 2.

Refer supplement: 1: for tobacco prevalence \& incidence of TRCs in Indian states.

\section{Discussion}

Many studies found out significant association between tobacco consumption and risk of following cancers- oral, mouth, tongue, head \& neck squamous cell cancer, hypopharynx, oropharynx, larynx, lung and esophagus. But to the best of our knowledge we could not find any prospective study that was conducted to decline tobacco related cancer incidence by reducing tobacco consumption. However, there had been studies on cancer mortality and tobacco habit reduction which we are trying to explore.

Dr. Pierce and his team compared smoking trends in California and other states from year 1974-2014 with the help of data from the National Health Interview Survey. They considered a representative 


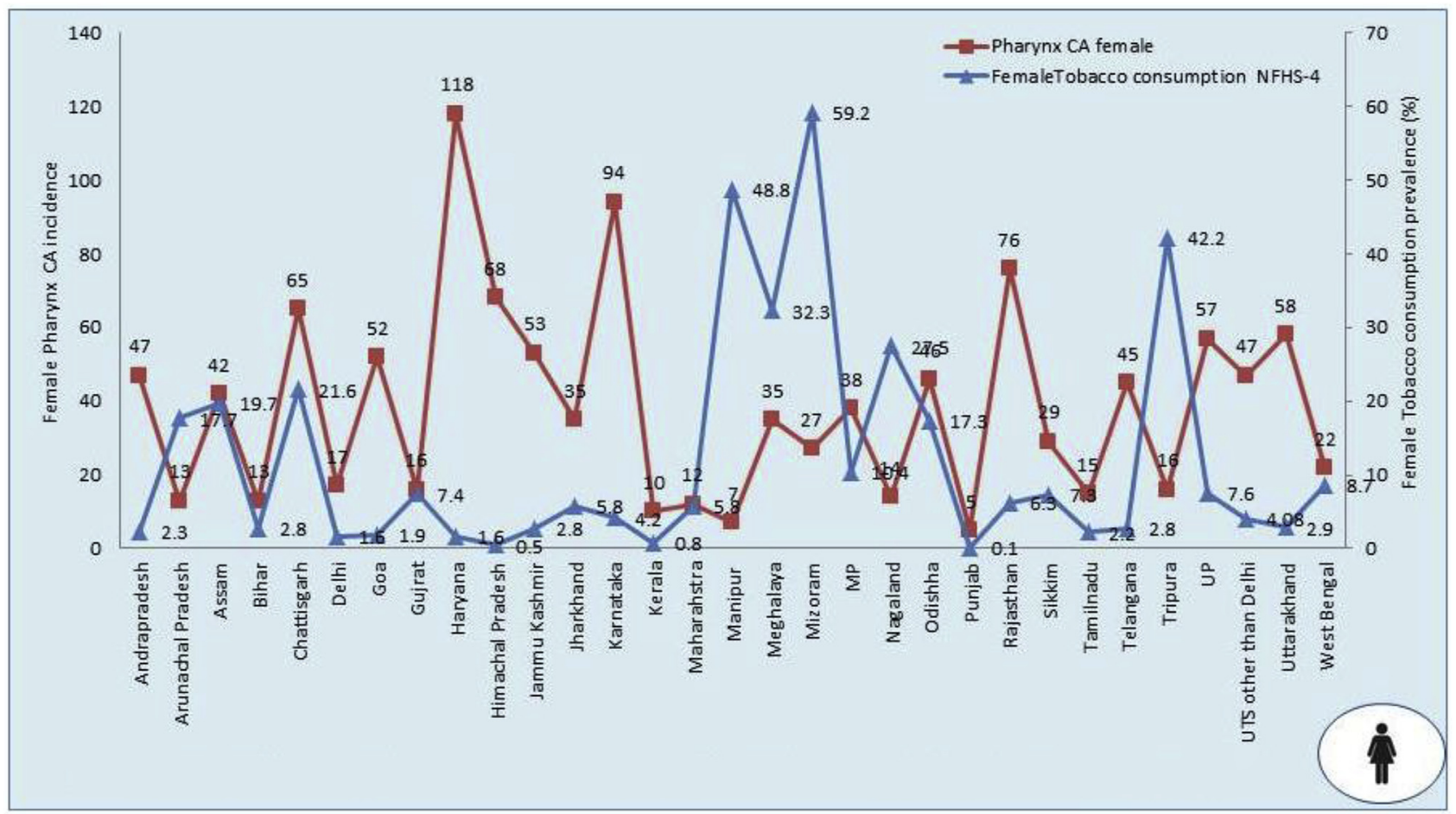

Fig. 9. Relationship between Tabacco consumption prevalence (\%) and pharynx CA insidence (per 10L pop) among Female.

sampling of 962,174 residents in the United States, approximately $10 \%$ of whom lived in California. The researchers found lung cancer mortality in California declined from an annual rate of 108 per 100,000 populations aged 35 years and older in 1985 to 62.6 per 100,000 in 2013, which was $33 \%$ faster than the rest of the United States. ${ }^{11}$ In 1985, lung cancer mortality rates for persons aged 35 years and older were similar between California and the rest of the country, but by 2013 rates in California were $28 \%$ lower (62.6 vs 87.5 per 100,000 population, respectively). This was a result of tobacco control policies working in favour of reduction of incidence \& mortality of lung cancer. ${ }^{11}$

John Pierce examined trends in smoking behaviour before, during \& after the California Tobacco Control Programme. As compared to the rest of United States, in California the annual rate of decline in monthly per capita consumption increased significantly by a factor of $52 \%$ during January 1989 through December 1993.The programme lost the

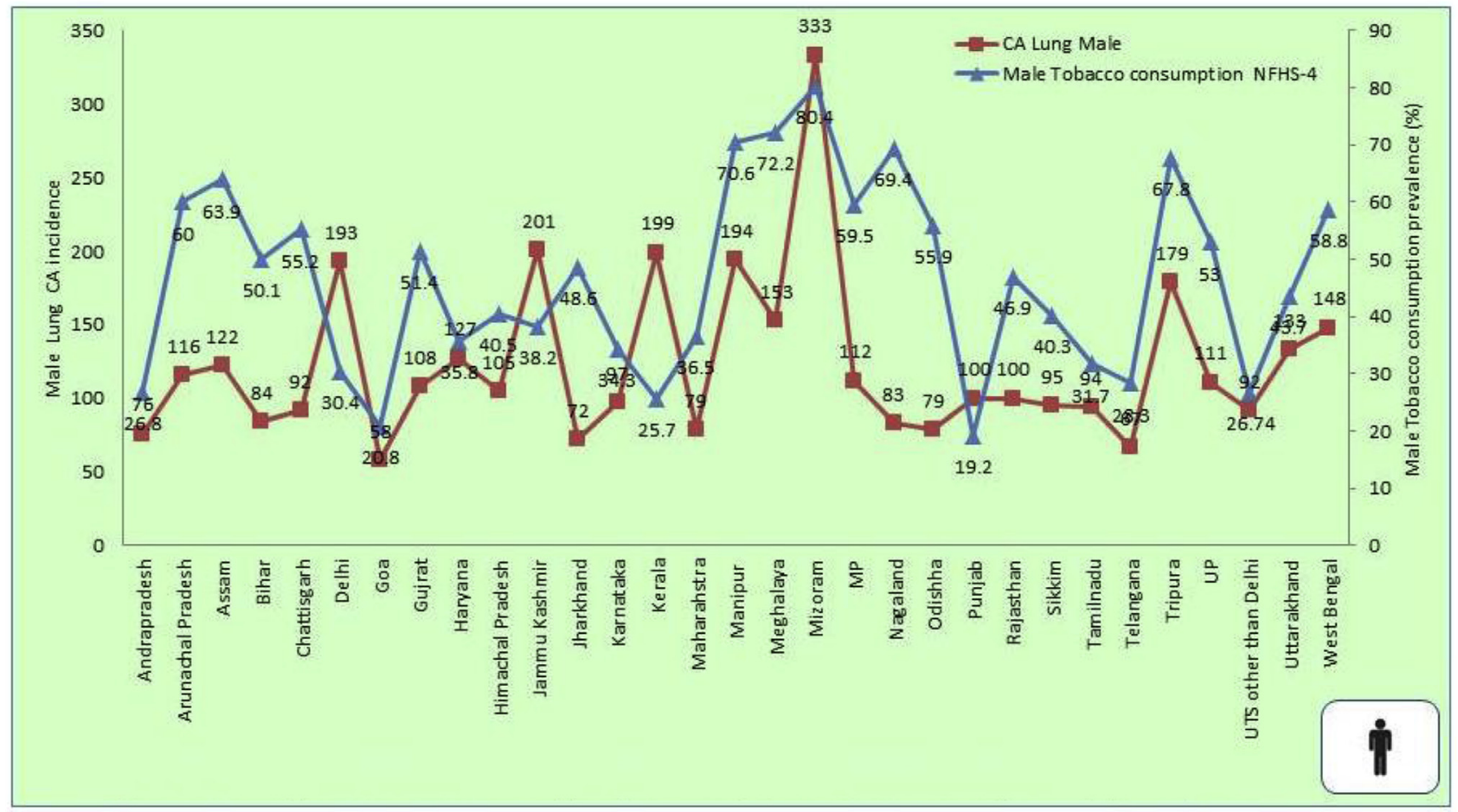

Fig. 10. Relationship between Tabacco consumption prevalence (\%) and Lung CA insidence (per 10L pop) among Male. 


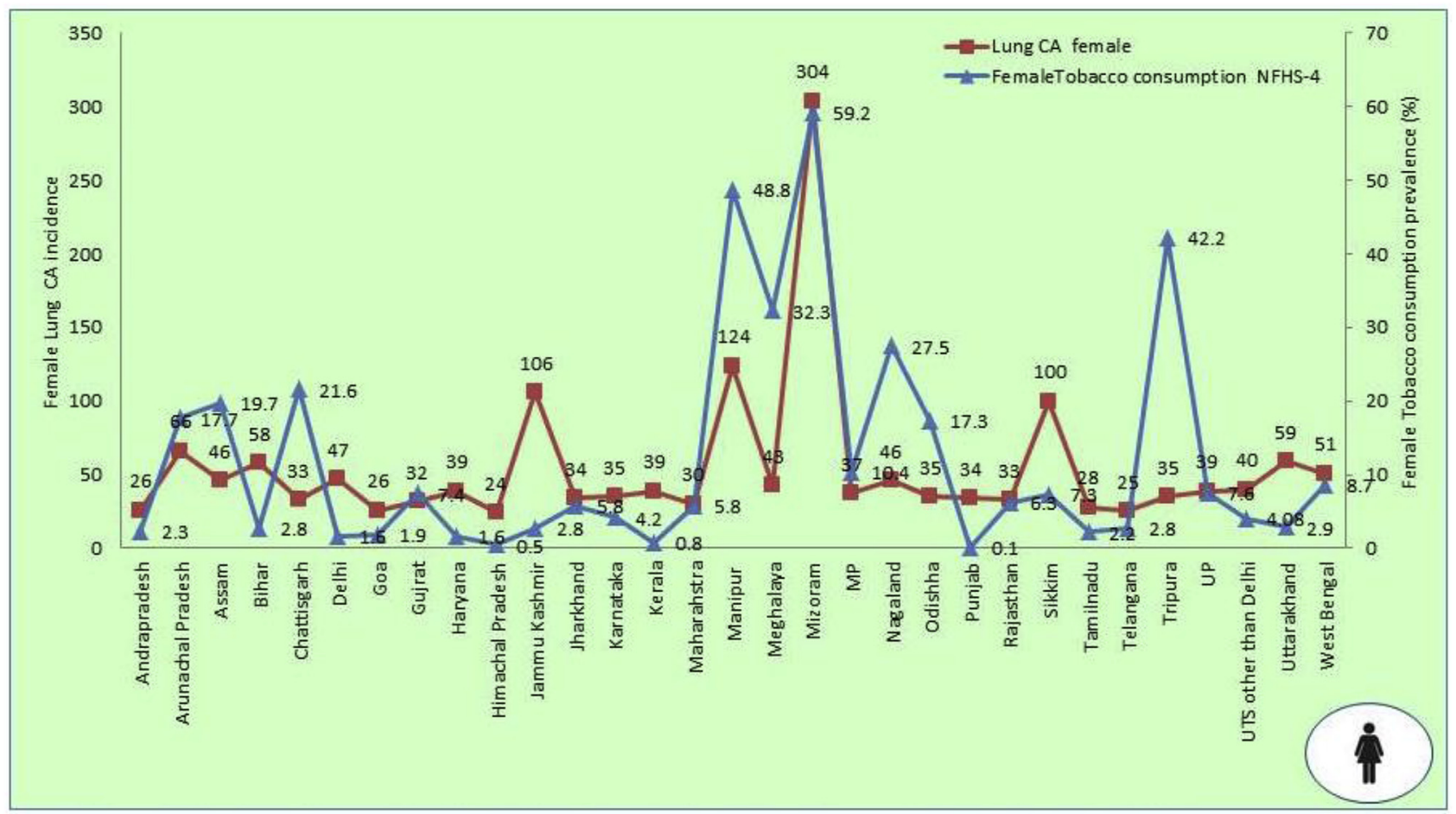

Fig. 11. Relationship between Tabacco consumption prevalence (\%) and Lung CA insidence (per 10L pop) among Female.

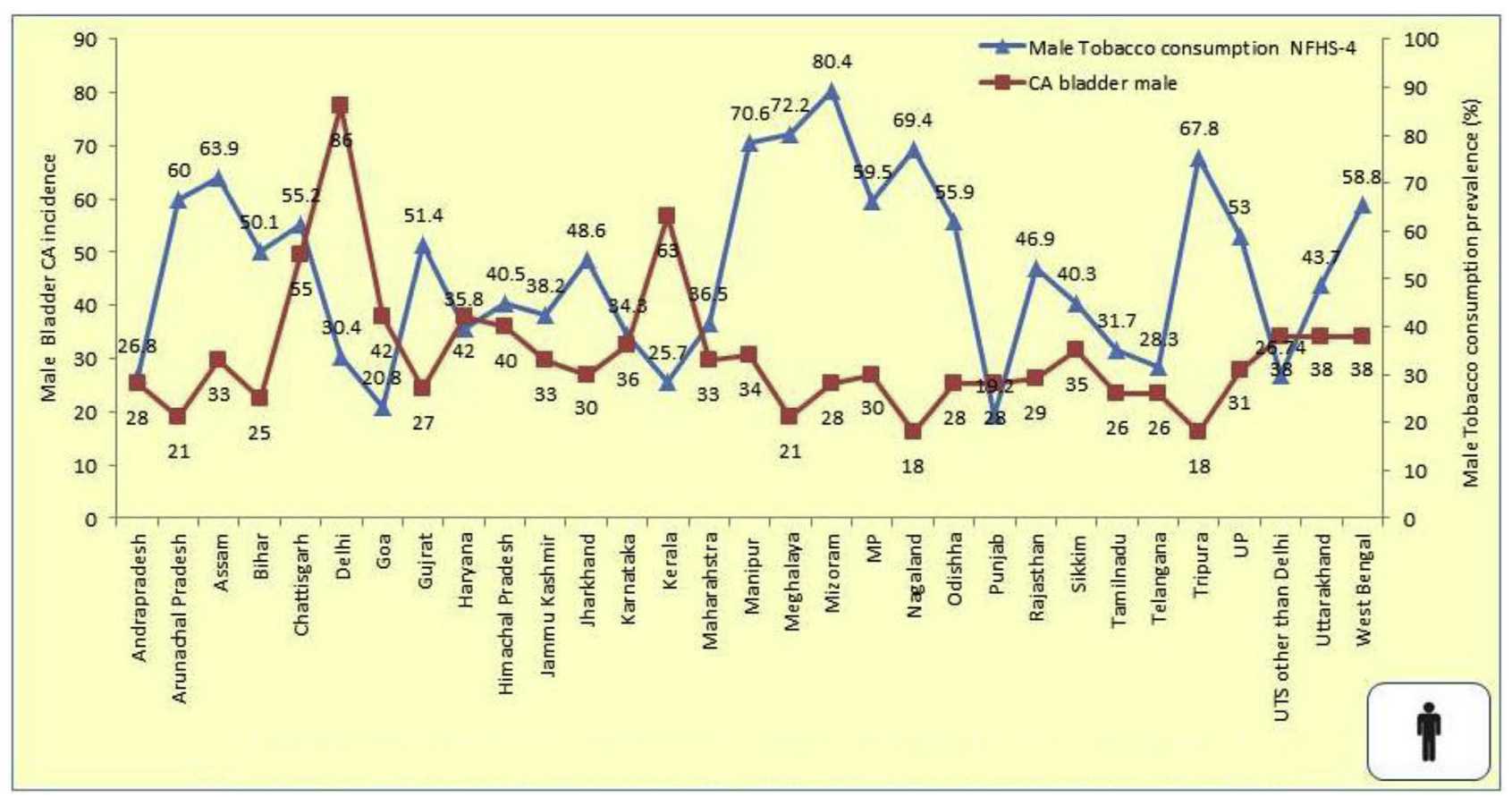

Fig. 12. Relationship between Tabacco consumption prevalence (\%) and Bladder CA insidence (per 10L pop) among Male.

success effect over a period of time. The possibilities could be due to lack of innovative approaches to interest population in tobacco control, or increased expenditures by the tobacco industry for advertising \& promotion. This can be a learning lesson for widespread dissemination of large health promotion programme. ${ }^{12}$

Tobacco control interventions included school programmes, increasing price through excise tax increases, large graphic warning labels on packages, restricting the tobacco industry's ability to advertise, tobacco control mass media programmes, smoke-free policies and restricting the ability of minors from purchasing tobacco products. The comprehensive community-wide programmes using multiple strategies had documented large declines for smoking initiation at Australia \& California. ${ }^{13}$ Pierce et al. described two case studies of Australia \& California. They were able to denormalise cigarette smoking and had a dramatic impact in reducing the proportion of adolescents who start to smoke. ${ }^{13}$

Luke Clancy proposed that properly applied restrictions can reduce teenage smoking as $85 \%$ of smokers begin their addiction in their teens. ${ }^{14}$ US Surgeon General, 2014a reports that there was evidence that continued smoking increases risk of a second primary cancer \& 


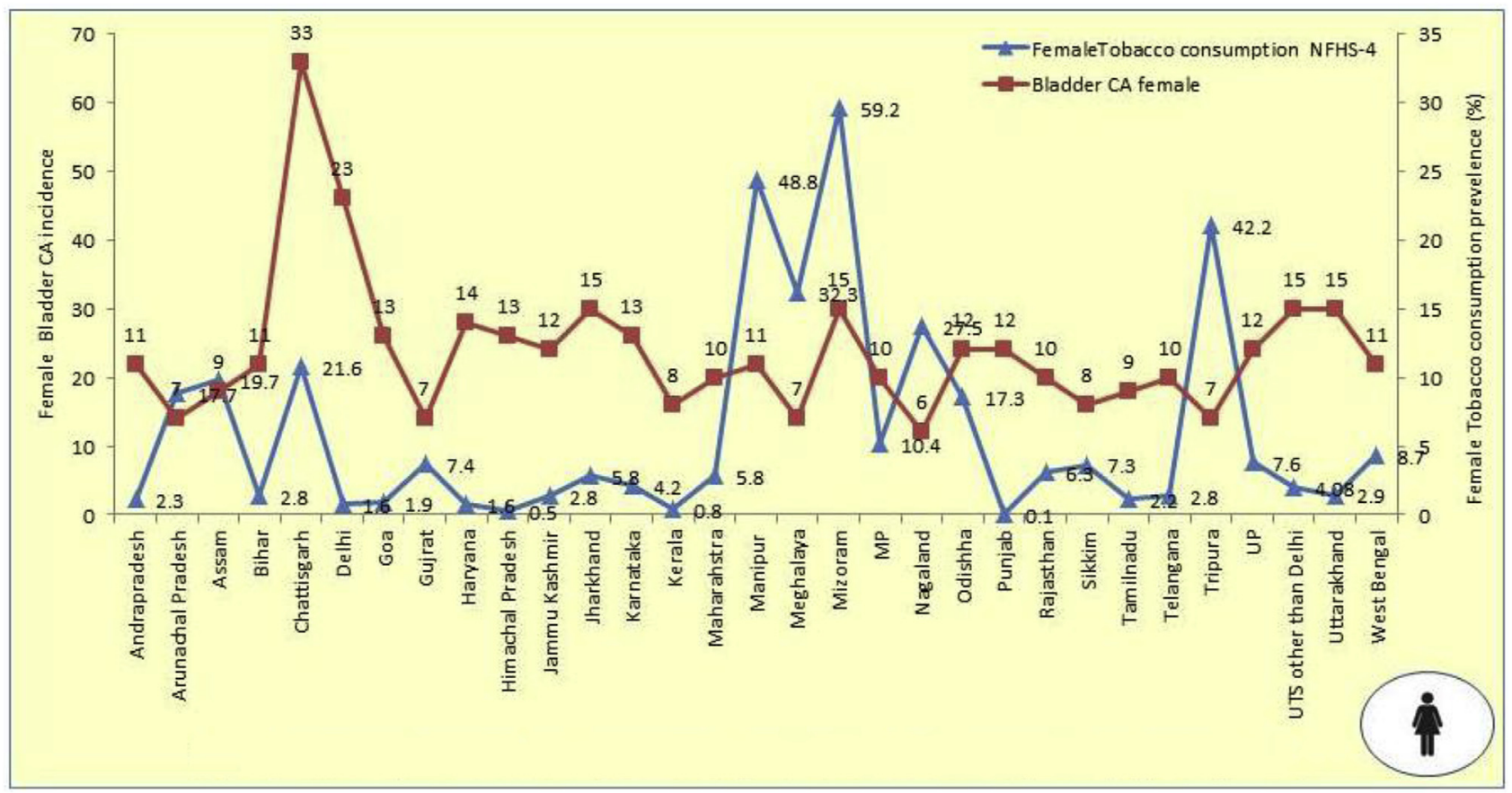

Fig. 13. Relationship between Tabacco consumption prevalence (\%) and Bladder CA insidence (per 10L pop) among Female.

Table: 2

Gender \& site wise linkage between tobacco prevalence and incidence rate of TRCs.

\begin{tabular}{lll}
\hline Type of TRCs & \multicolumn{2}{l}{$\begin{array}{l}\text { Reduction in Age standardised Incidence rate (per 10,00,000 } \\
\text { pop.) of TRCs, if 1\% reduction of tobacco prevalence is expected }\end{array}$} \\
\cline { 2 - 3 } & Male & Female \\
\hline Lip \& oral cavity & 0.038 & 11.78 \\
Larynx & 14.50 & 11.78 \\
Nasopharynx & 1.77 & 2.58 \\
Pharynx & 25.31 & 7.51 \\
Lung & 7.23 & 2.35 \\
Bladder & 1.37 & 0.91 \\
\end{tabular}

recurrence. ${ }^{15} \mathrm{M}$ Nichter gave an overview of Project Quit Tobacco International in four phases and explained that if adult tobacco consumption were to decrease by $50 \%$ by the year 2010 , approximately 180 million tobacco related deaths could be avoided. ${ }^{16}$ Manuela Marron ${ }^{17}$ et al. studied five cohort \& 40 case control studies which they explored that there was a reduction of head \& neck cancer risk after the cessation of tobacco smoking to the order of $16-85 \%$. They also pooled data from 18 case control studies from around the world within the International Head \& Neck Caner Epidemiology (INHANCE) Consortium to estimate the number of years of quitting required to observe a reduced risk \& whether the risk declines to the level of never smokers \& never drinkers. They concluded that the risk of head and neck cancer had decreased significantly among persons who had stopped tobacco smoking 1-4 years previously. Current and former tobacco smokers had a higher relative risk of laryngeal cancer than oral or pharyngeal cancer. These findings are in agreement with our study. Reduction of tobacco consumption has a public health implication and quitting/ stopping initiation of this habit can be encouraged by public health interventions. ${ }^{17}$ A retrospective study conducted in UK by C Koshiaris ${ }^{18}$ et al. observed quitting smoking reduces the risk of death in lung cancer and may do so in Upper aero-digestive tract (UAT) cancer but there was no evidence that quitting smoking can reduce bladder cancer. There is stronger evidence that quitting among diagnosed cancer can improve cancer outcomes in UAT and lung cancer. ${ }^{18}$ Our study had similar findings that of C Koshiaris.

Jagdish Kaur, D.C. Jain recommends effective tobacco control was dependent on balanced implementation of demand and supply reduction strategies by the Government. This comprised inter-sectoral coordination involving stakeholder departments and ministries ${ }^{19} \mathrm{We}$ also suggest similarly.

Limitations: NFHS-IV refers to data for tobacco prevalence was conducted between the age group of 15-49 years. The type and amount of tobacco consumption were directly associated with incidence of tobacco related diseases and mortality. But NFHS -IV data do not collect data on type \& amount of tobacco consumed which was the weakness of NFHS apart from the age group considered in NFHS -4 \& GBD study. The Global Burden of Disease study had limitations in terms of approach that was considered for estimation of age adjusted Incidence rate of cancers from all the age groups. Cancer Data was collected from cancer registry, vital registration systems-cause of death, SRS-verbal autopsy from all states of India. Absence of population based cancer registry in large populous states, sparse presence of rural cancer registries, inadequate coding, misclassification during cause of death certification are few limitations of GBD study.

\section{Conclusion}

The linkage explored in this study between tobacco consumption \& TRCs incidence consistently notifies direct relation between the two. One unit reductions of tobacco prevalence may decline incidence of lip, oral cavity and larynx cancer among female noticeably while reduction of tobacco will give remarkable results for pharynx cancer among Indian males. Apart from various types, ways of tobacco consumption, other factors predispose an individual for that particular type of cancer. They are geographical variation, genetic, cultural, dietary, lifestyle etc. The level of education and poverty has important role in availing the health care facility \& compliance to disease treatment. $92 \%$ of unorganised work place in rural \& urban parts of India needs to be addressed for worksite promotion ${ }^{20}$ of tobacco cessation, individual \& group counseling. Tobacco, notorious agent for cancers, must be banned to eradicate the prevalence of tobacco related cancers. More than quarter of cancers in India can be prevented if tobacco use is 
banned. Reduction in tobacco consumption should include prevention of initiation and promote cessation among the young population, disadvantaged groups, unorganised sector of workplace. Integration of tobacco control activities with developmental programmes is desirable like poverty alleviation, rural developmental schemes, women and child development, tribal welfare programme, trained professionals at Tobacco cessation clinics all over. Apart from comprehensive bans ${ }^{21}$ on tobacco advertising, promotion and sponsorship, specific interventionssuch as narrating \& broadcasting the stories of affected individuals is imperative to diminish incidence of TRCs. Comprehensive bans on tobacco advertising, promotion and sponsorship can reduce premature deaths due to tobacco consumption will prevent youth epidemic of tobacco. But one has to remember, blanket ban on tobacco is involved with complex economic situation in India, rehabilitation concern of workers involved in tobacco industries, alternate crop production.

We need to plan holistic, coercive and systematic approach and implement it vigorously to restrict the burden of TRCs. Multipronged strategy, multi-sectoral approach involving not only political will \& support but public private partnership and community participation can decline tobacco consumption. Capacity building and training of existing public health resource, improved infrastructure for early detection, diagnosis and treatment of TRCs can curtail the burden related to it.

\section{Funding}

There was no funding support for this work.

\section{Declaration of competing interest}

None.

\section{Appendix A. Supplementary data}

Supplementary data to this article can be found online at https:// doi.org/10.1016/j.cegh.2020.04.029.

\section{References}

1. Farhood Bagher, et al. Incidence and mortality of various cancers in Iran and compare to other countries: a review article. Iran J Public Health. 2018 Mar;47(3):309-316.

2. Globocan 2018: counting the toll of cancer. Edit Lancet. 2018 Sept:392.

3. Mallath Mohandas, et al. The growing burden of cancer in India: Epidemiology \& social context. Lancet. 2014 April:1-8.

4. Dr. Poonam Khetrapal Singh. Media Statement on World Cancer Day 2016. WHO South East Asia. Available from: http://www.searo.who.int/mediacentre/features/ 2016/world-cancer-day-2016/en/ (accessed on 7/9/2019).

5. Asthana Smita, Patil Rakshit S, Satyanarayana Labani. Tobacco-related cancers in India: a review of incidence reported from population-based cancer registries. Indian J Med Paediatr Oncol. 2016 Jul-Sep;37(3):152-157.

6. Malhotra RK, et al. Trends and future burden of tobacco: related cancers incidence in Delhi urban areas: 1988-2012. Indian J Publ Health. 2019;63(1):33-38 Jan-March.

7. Rani M, Bonu S, Jha P, Nguyen SN, Jamjoum L. Tobacco use in India: prevalence \& predictors of smoking \& chewing in a national cross sectional household survey. Tobac Contr. 2003;12:e4.

8. Prakash C. Gupta. Tobacco related cancer - its impact on the health economy. Health Adm. Vol: XVII, Number 1: 85-92.

9. India State-Level Disease Burden Initiative Cancer Collaborators. The burden of cancers and their variations across the states of India: the Global Burden of Disease Study 1990-2016. Lancet. 2018 Oct;19:1289-1306.

10. http://rchiips.org/nfhs/factsheet_nfhs-4.shtml, Accessed date: 7 September 2019.

11. Fillon Mike. Tobacco control initiatives cut the number of lung cancer deaths in California by 28\%. Perspectives: research in context. 2019 March-April;69(2):83-85.

12. Pierce John P, et al. Has the California tobacco control program reduced smoking? $J$ Am Med Assoc. 1998;280(10):893-899.

13. John P Pierce Victoria M White, Emery Sherry L. What public health strategies are needed to reduce smoking initiation? Tobac Contr. 2012;21:258-264.

14. Clancy Luke. Reducing lung cancer and other tobacco-related cancers in europe: smoking cessation is the key. Oncol. 2014;19:16-20.

15. Warren Graham W. The 2014 Surgeon general's report: "the health consequences of smoking-50 Years of progress": a paradigm shift in cancer care. Cancer. 2014 July 01;120(13):1914-1916.

16. Nichter M. Introducing tobacco cessation in developing countries: an overview of project quit tobacco international. Tobac Contr. 2006;15(Suppl I):i12-i17.

17. Marron Manuela, et al. Cessation of alcohol drinking, tobacco smoking and the reversal of head \& neck cancer risk. Int J Epidemiol. 2010;39:182-196.

18. Koshiaris C, et al. Smoking cessation and survival in lung, upper aero-digestive tract and bladder cancer: cohort study. Br J Canc. 2017;117:1224-1232.

19. Kaur Jagdish, Jain DC. Tobacco control policies in India: implementation and challenges. Indian J Publ Health. 2011 July-Sept;55(3):220-227.

20. Mohan Priya, Lando Harry A, Panneer Sigamani. Assessment of tobacco consumption and control in India. Indian J Clin Med. 2018;9:1-8.

21. Arora Monika, Nazar Gaurang P. Prohibiting tobacco advertising, promotions \& sponsorships: tobacco control best buy. Indian J Med Res. 2013 May;137(5):867-870. 